\title{
Preparation, Physicochemical Properties, Invitro And In Vivo Release Evaluation of Chitosan Decorated Curcumin Loaded Niosome
}

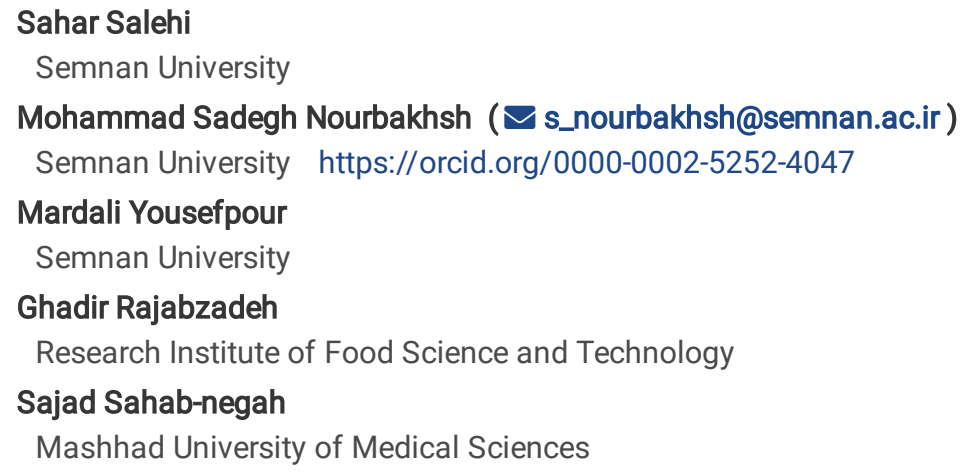

Research Article

Keywords: Niosome, Chitosome, Curcumin, Drug Delivery System, Blood -brain barrier

Posted Date: May 6th, 2021

DOI: https://doi.org/10.21203/rs.3.rs-429869/v1

License: (c) (1) This work is licensed under a Creative Commons Attribution 4.0 International License. Read Full License 


\section{Abstract}

Purpose: Based on several pharmacological activities of curcumin (Cn), it has been introduced as an ideal candidate for different neurological disorders. But poor solubility, rapid clearance and low stability have limited its clinical application. Development of curcumin loaded smart niosome for crossing blood brain barrier could be and interesting option to overcome these limitations.

Object: The aim of this study is preparation and characterization of chitosan decorated curcumin loaded niosome(CH-CLN) and evaluation of invitro release and in vivo bioavailability and bio stability of curcumin in the brain tissue.

Methods: Niosomal formulations were prepared by modified heating method by using 3 -factor and 3 level mixture design. The formulations were characterized using DLS, zeta potential, Fourier transform infrared (FTIR) spectroscopy, high performance liquid chromatography (HPLC) and transmission electron microscopy(TEM). The entrapment efficiency and invitro release were also evaluated. Wistar rats were subjected to intraperitoneal injection (i. p.) of formulations, and curcumin 15 minutes before perfusion. Cn concentration in different parts of the central nervous system, liver, and plasma was analyzed for in vivo analysis.

Results: Encapsulation efficiency was obtained 75\% for optimized formulation and presented sustain release, followed by the Hixon-Crowell model. The particle size was about $100 \mathrm{~nm}$ with polydispersity index (PDI) of 0.2. Chitosan decorated niosomal formulation increased $\mathrm{Cn}$ concentration in central nervous system.

Conclusion: We can conclude the chitosan decorated curcumin loaded niosome improved bioavailability of curcumin in brain tissue and cold be a promising tool for crossing blood-brain barrier

\section{Introduction}

Curcumin [1,7-bis (4-hydroxy-3-methoxyphenyl)-1,6-heptadiene-3,5-dion], as the major component of turmeric, has been extremely used for curing different kinds of cancer cells such as breast, prostate, bone, and neck cancers, as well as several chronic neurological diseases such as Alzheimer[1-3]. Moreover, it can also be used due to its anti-inflammatory, antioxidant, antimicrobial, anti-amyloid, anti-cystic fibrosis, anti-proliferative properties, and as a wound healing agent[4,5].

Although $\mathrm{Cn}$ is claimed to be promising in numerous therapeutic interventions, it has demonstrated a poor bioavailability due to its poor aqueous solubility and a low stability against alkaline $\mathrm{pH}$ conditions. More importantly, $\mathrm{Cn}$ exhibits an extensive first-pass metabolism, which is known as the main reason for the limitation of the retention time in the body. Accordingly, a designed carrier system could considerably increase the range of its achievable pharmaceutical applications[6.7].

In the last two decades, different delivery systems containing $\mathrm{Cn}$ have been developed; for instance, cyclodextrin; polymeric microparticles; and lipid systems such as lipid nanoparticles, niosome, liposomes, nanostructured lipid carriers, and nanoemulsions[7- 11]. The delivery system not only solves the mentioned Cn downside, but it also increases permeability via the BBB for brain drug delivery.

Noticeable attention has been drawn to niosomes stemming from their exceptional stability and exemplary property, as loaded by hydrophilic or hydrophobic molecules. Niosome can overcome the enhance the bioavailability of $\mathrm{Cn}$ via increasing its retention time in the blood stream and passing the BBB[12.13]. Moreover, there have been many efforts to improve the stability, and target delivery and pharmacokinetic behavior of niosomes. In this regard, different materials such as polyethylene glycol, hyaluronic acid, antibodies, and chitosan were utilized for the reformation of the niosme surface. Accordingly, chitosan[ $\beta-(1-4)$-linked d-glucosamine (deacetylated unit) and $\mathrm{N}$-acetyl-d-glucosamine (acetylated unit)] are the most frequently utilized polymers in the formulation of coated niosomes(Khalifa and Abdul Rasool 2017).

In the same context, Mythri et al. (2007) showed that retention time of $\mathrm{Cn}$ in rat serum extended by the use of Cn-phospholipid(Mythri et al. 2007).Previously, Chirio et al. (2018) formulated Cn loaded CLN intending to achieve the prolonged permanence of the drug in the bloodstream(Chirio et al. 2018). On the other side Recently, Khalaf et al. (2019) in their study have demonstrated that the chitosan coated niosome increased Olanzapine brain targeting compared to bare niosome(Khallaf et al. 2019).

To the best of our knowledge, $\mathrm{Cn}, \mathrm{CLN}$, and CH-CLN distributions in the brain, plasma, and liver of Wistar rat have not been reported so far. Quantity of $\mathrm{Cn}$ in the target site is considered as an essential factor for clarifying the therapeutic effects of the drug in those organs. Hence, in this study, we presented the chitosan coated niosome as $\mathrm{Cn}$ carrier, to validate the hypothesis that $\mathrm{CH}-\mathrm{CLN}$ could target the brain and increase the BBB permeability, which is distributed at different regions of brain, liver, and plasma.

\section{Material And Methods}




\subsection{Materials}

Polysorbate 60 (Tween ${ }^{860)}$ ), Sorbitan monostearate (Span®60), Dicetyl phosphate (DCP), Chitosan (Low molecular weight), cholesterol (Chol), heparin, dialysis bag (MWCO = 12000 Da (, and Cn were purchased from Sigma-Aldrich (Germany). Moreover, Absolute ethanol, glycerol, carboxymethylcellulose, and methanol were purchased from Merck (Germany). All these materials were analytically graded. Normal saline was obtained from Shahid Ghazi (Iran). Ketamine and Xylazine were obtained from Alfasan (Netherland).

\subsection{Mixture Design (M.D.)}

In this study, with the aim of altering the formulations, M.D. with three-level, three-factor and seven experimental runs were adopted. Table 1 shows the independent and dependent variables as well as their levels.

Table 1

The independent and dependent variables of MD with statistical

parameters.

\begin{tabular}{|llll|}
\hline Independent Variables (Factors) & Levels & \multirow{2}{*}{ Units } \\
\cline { 2 - 3 } & Low & High & \\
\hline Span 60 concentration & 0.06 & 0.28 & $\mathrm{G}$ \\
\hline Tween 60 concentration & 0.06 & 0.28 & $\mathrm{G}$ \\
\hline Chol concentration & 0.06 & 0.28 & $\mathrm{G}$ \\
\hline Dependent variables (Response) & Units & & Goal \\
\hline Mean vesicle size & $\mathrm{Nm}$ & & Minimize \\
\hline Poly disperse index (PDI) & Value & Minimize \\
\hline Zeta potential & $\mathrm{mV}$ & Maximize \\
\hline Entrapment & $\%$ & & Maximize \\
\hline
\end{tabular}

\section{3. Vesicle preparation}

Different niosome formulations, (Table 2), were synthesized in terms of the heating method explained by Mozaffari (2008) (Mozafari et al. 2008) and Rajabzadeh (2017)(Basiri et al. 2017a) with some modifications. Briefly, to hydrate the appropriate amounts of surfactants applying phosphate- buffered saline (PBS, pH 7.4, autoclaved), they were stirred for 60 min at $25^{\circ} \mathrm{C}$. Afterward, the Chol dispersion was vigorously stirred for $30 \mathrm{~min}$ at $120^{\circ} \mathrm{C}$ on a hotplate.

Table 2

MD in the process of niosome preparation and physicochemical characteristics of $\mathrm{Cn}$ loaded noisome (mean $\pm \mathrm{SD}, \mathrm{n}=3$ ).

\begin{tabular}{|c|c|c|c|c|c|c|c|}
\hline \multirow[t]{2}{*}{ Sample } & \multicolumn{3}{|c|}{ Independent Variables } & \multicolumn{4}{|c|}{ Dependent variables } \\
\hline & $\begin{array}{l}\text { Span } \\
60\end{array}$ & $\begin{array}{l}\text { Tween } \\
60\end{array}$ & Cholesterol & $\begin{array}{l}\text { Z average } \\
(\mathrm{nm})\end{array}$ & PDI (value) & $\begin{array}{l}\text { Zeta } \\
\text { potential } \\
(\mathrm{mV})\end{array}$ & $\mathrm{EE} \%(\mathrm{Cn})$ \\
\hline NHM1000 & 0.134 & 0.133 & 0.133 & $111 \pm 0.32$ & $\begin{array}{l}0.188 \pm \\
0.002\end{array}$ & $-30 \pm 0.8$ & $75 \pm 0.458$ \\
\hline NHM1100 & 0.206 & 0.097 & 0.097 & $128 \pm 0.32$ & $\begin{array}{l}0.251 \pm \\
0.015\end{array}$ & $-26 \pm 0.2$ & $33 \pm 0.1$ \\
\hline NHM1200 & 0.06 & 0.28 & 0.06 & $70 \pm 0.44$ & $0.21 \pm 0.01$ & $-23 \pm 0.1$ & $88 \pm 0.42$ \\
\hline NHM1300 & 0.097 & 0.201 & 0.097 & $97 \pm 0.32$ & $\begin{array}{l}0.182 \pm \\
0.002\end{array}$ & $-26 \pm 0.1$ & $60 \pm 0.22$ \\
\hline NHM1400 & 0.097 & 0.097 & 0.206 & $153 \pm 0.43$ & $\begin{array}{l}0.37 \pm \\
0.001\end{array}$ & $-27 \pm 0.3$ & $90 \pm 0.23$ \\
\hline NHM1500 & 0.28 & 0.06 & 0.06 & $117 \pm 0.43$ & $\begin{array}{l}0.30 \pm \\
0.003\end{array}$ & $-36 \pm 0.2$ & $64 \pm 0.31$ \\
\hline NHM1600 & 0.06 & 0.06 & 0.28 & $125 \pm 0.46$ & $\begin{array}{l}0.140 \pm \\
0.001\end{array}$ & $-30 \pm 0.6$ & $32 \pm 0.12$ \\
\hline
\end{tabular}


The obtained colloid was added to a preheated $\left(5 \mathrm{~min}, 60^{\circ} \mathrm{C}\right)$ mixture of $\mathrm{Cn}(100 \mu \mathrm{M}($ Sharma et al. $2015 \mathrm{~b}))$, glycerol (final concentration of $3 \%$, v/v), DCP (DCP: Surfactant, 0.1 molar ratio) and ethanol ( $1 \mathrm{cC}$ ), which were stirred ( at $60^{\circ} \mathrm{C}, 1000 \mathrm{rpm}$ ) on a hotplate stirrer (IKA®C. MAG HS7 Safety Control, IKA, Malaysia) for $60 \mathrm{~min}$. The reaction was performed under a nitrogen atmosphere in a handmade glass vessel introduced by Mozaffari. Subsequently, sonication of the sample was performed using a probe sonicator (Sonopuls HD-3100, BANDELIN electronic GmbH \& Co. Germany) for $16 \mathrm{~min}$ (180 sec "pulse on" and $30 \mathrm{sec}$ "pulse off"). After the preparation of the loaded niosome, they were kept for 30 min at room temperature.

\subsection{Preparation of Chitosan Coated CLN (CH-CLN).}

The $\mathrm{CH}$-CLNs were prepared according to Marianecci with some modifications(Rinaldi et al. 2020). At first, $\mathrm{CH}$-CLNs were made by the addition of optimum formulation of $\mathrm{CLNs}$ into $\mathrm{CH}$ solution $(1: 2, \mathrm{v} / \mathrm{v})$. The mixture was then stirred for $60 \mathrm{~min}$ at $25^{\circ} \mathrm{C}$. Thereafter, the obtained mixture was adjusted to $\mathrm{pH} 4.5(\mathrm{NaOH}, 1 \mathrm{M})$, and then sonication of the sample was performed using a probe sonicator for $5 \mathrm{~min}$ (180 sec "pulse on" and $30 \mathrm{sec}$ "pulse off).

\subsection{Cn Determination}

The $\mathrm{Cn}$ measurement was performed at $40^{\circ} \mathrm{C}$ on a High-performance liquid chromatography (Waters, USA). The HPLC system contained a Waters 1525 binary pump, and $2489 \mathrm{Uv}$-Vis detectors at $420 \mathrm{~nm}$. Also, a reversed-phase Inertsustian Swift C18 column $(4.6 \times 250 \mathrm{~mm}$, particle size $5 \mu \mathrm{m}$ ) was used for HPLC analysis. The mobile phase was composed of $5 \%$ acetonitrile solution in water buffered to $\mathrm{pH} 2.7 \mathrm{by} 10 \%$ ortho-phosphoric acid $(90: 10 \mathrm{v} / \mathrm{v})$ at a flow rate of $1 \mathrm{ml} / \mathrm{min}$.

The stock solution was prepared by the dissolution of $5 \mathrm{mg} \mathrm{Cn}$ in $2.5 \mathrm{ml}$ of methanol $(2000 \mu \mathrm{g} / \mathrm{mL})$. The obtained solution was then diluted by the mobile phase to make a serial concentration of the working standard solution $(0.4,0.8,1.2,1.6,2.0$, and $2.4 \mu \mathrm{g} / \mathrm{mL})$. All the solutions were stored at $4^{\circ} \mathrm{C}$ in amber glassware and then sonicated for $15 \mathrm{~min}$ before usage.

The limit of detection (LOD) and the limit of quantification (LOQ) were 0.186712 and 0.565794 , respectively.

\subsection{Encapsulation efficiency}

CLN and CH-CLN suspensions ( $5 \mathrm{ml}$ ) were loaded in a centrifuge Amicon MPS (Millipore, USA) filtration tube and then centrifuged at $6000 \mathrm{rpm}$ (Hettich centrifuge, model EBA 20, Germany) to separate niosomes from the unloaded $\mathrm{Cn}$. In this regard, the following equation was used to calculate the Cn encapsulation efficiency(Moghddam et al. 2016).

$\mathrm{EE} \%=($ total $\mathrm{Cn}-\mathrm{Cn}$ in supernatant) $/($ total $\mathrm{Cn}) \times 100$

2.7 Size, polydispersity index and zeta potential analysis

The sample sizes, polydispersity index (PDI) and zeta potential of diluted CLN and CH-CLN (1:10 v/v) were measured using dynamic light scattering method (DLS) on Zeta sizer Nano ZS (Malvern Instruments Ltd., United Kingdom) by a helium-neon laser at $630 \mathrm{~nm}$ at room temperature(Kassem et al. 2017).

2.9. Fourier Transform Infrared (FT-IR) spectroscopy

The functional groups of the samples' components were studied by FT-IR (Thermo Nicolet, AVATAR, 370 FT-IR, USA). The samples were scanned with a resolution of $4 \mathrm{~cm}^{-1}$ between $4000 \mathrm{~cm}^{-1}$ and $400 \mathrm{~cm}^{-1}$.

\subsection{Transmission electron microscopy (TEM)}

TEM image of CLN and CH-CLN have been taken by utilized 2\% uranyl acetate solution as a staining agent (Leo 912 Omega TEM ,Germany).

\subsection{3. $\mathrm{Cn}$ release studies}

The release of $\mathrm{Cn}$ was accomplished using the dialysis technique(Le and $\mathrm{Kim}$ 2019), (Xu et al. 2016). $5 \mathrm{~mL}$ of sample were wrapped in a dialysis bag (D0666, Sigma). In order to immerse the dialysis bag, $50 \mathrm{~mL}$ of a gastric fluid (SGF, HCl solution $0.1 \mathrm{~N}, \mathrm{pH}=1.2$ ) and intestinal fluid (SIF, PBS, pH 7.4) have been simulated for 2 and $6 \mathrm{~h}$ respectively in a digital shaker incubator (Hanyang, SI-64A, $50 \mathrm{rpm}, 37^{\circ} \mathrm{C}$ ). Accordingly, both matrices contained $0.1 \%(\mathrm{v} / \mathrm{v})$ Tween 80 .

To evaluate the kinetic behavior and release mechanism, the result derived from release studies was fitted into different mathematical equations as demonstrated in Table 4. 
Table 4

In vitro release of $\mathrm{Cn}$ in niosome and chitosan

coated niosome R2 and k values.

\begin{tabular}{|lllll|}
\hline Function & $\mathrm{R}^{2}$ & \multicolumn{3}{c|}{$\mathrm{K}$} \\
\cline { 2 - 5 } & $\mathrm{CLN}$ & $\begin{array}{l}\text { CH- } \\
\text { CLN }\end{array}$ & CLN & $\begin{array}{l}\text { CH- } \\
\text { CLN }\end{array}$ \\
\hline Zero order & 0.88 & 0.88 & 5.75 & 1.57 \\
\hline First order & 0.92 & 0.92 & 0.125 & 0.6 \\
\hline Higuchi & 0.95 & 0.97 & 16.25 & 4.44 \\
\hline $\begin{array}{l}\text { Hixson- } \\
\text { Crowell }\end{array}$ & 0.96 & 0.90 & 0.44 & 0.02 \\
\hline
\end{tabular}

\subsection{Animal study}

2.14.1 Animals

Wistar rats (12 male individuals, $190 \mathrm{~g} \pm 10 \mathrm{~g}$ in body weight) were obtained from house breeding colonies at the Neuroscience Department of Mashhad University of Medical Sciences for drug control. Afterward, they were kept under standard conditions (12h light and $12 \mathrm{~h}$ dark cycle, $23 \pm 1^{\circ} \mathrm{C}$, with easy access to food and water sources). Animal handling and all the other related procedures were approved by Medical Sciences, Ethical Committee Acts (Mashhad, Iran, IR.mums.Rec.1399.287). The minimum number of animals was used to respect animal rights.

2.14.2 Drug administration and accessing its bioavailability

The rats were randomly divided into the following three groups (4 rats in each group): (1) treated with free Cn (group I), (2) treated with CLN (group II), and (3) treated with $\mathrm{CH}-\mathrm{CLN}$ (group III).

0.5\%w/v aqueous solution of carboxymethylcellulose (CMC) was then applied for preparing free Cn solution(Hoppe et al. 2013).

Subsequently, the $50 \mathrm{mg} / \mathrm{kg}$ of freshly $\mathrm{Cn}$ suspension, CLN, and $\mathrm{CH}$ - CLN were administered by intraperitoneal injection (i.p.) to the rats(Tsai et al. 2011). By passing 15 minutes from the injection, the experimental rats were anesthetized by a mixture containing $10 \%$ ketamine (100 $\mathrm{mg} / \mathrm{kg}$ body weight) and $2 \%$ xylazine ( $30 \mathrm{mg} / \mathrm{kg}$ body weight) (Tiwari et al. 2014). After anesthetizing, the cardiac puncture was utilized to collect $400 \mu$ blood with a heparinized syringe. Thereafter, the rats were perfused with normal saline to remove their brain and liver. Moreover, to evaluate crossing the BBB, the cerebral cortex, hippocampus, cerebellum, and striatum were collected.

Afterward, the samples were stored in microtubes at $-80^{\circ} \mathrm{C}$. Cn was extracted from plasma, and different regions of the brain and liver according to the protein precipitation technique proposed by Ravi (2018) with some modifications(Dalvi et al. 2018). Briefly, $400 \mu \mathrm{L}$ methanol was added to $90 \mu \mathrm{L}$ Plasma. The mixture was then vortexed for $2 \mathrm{~min}$. Subsequently, the sample was centrifuged at $14000 \mathrm{~g}$ for $20 \mathrm{~min}$ at $4^{\circ} \mathrm{C}$. Following that, the supernatant was dried under $\mathrm{N}_{2}$ gas at $40^{\circ} \mathrm{C}$. Finally, $100 \mu \mathrm{L}$ mobile phase was added for reconstitution, which was then analyzed by high-performance liquid chromatography (HPLC, Waters equipped with pump1525 binary pump along with UV2485and FLD2475 detector, USA).

Different regions of brain and liver tissues were homogenized using a probe sonicator for $1 \mathrm{~min}(25 \mathrm{son} 15 \mathrm{~s}$ off $)$ at $4^{\circ} \mathrm{C}$ in $\mathrm{PBS}(1: 4 \mathrm{~W} / \mathrm{V}, \mathrm{pH}$ : 7.4). Next, the mixtures were centrifuged at $14000 \mathrm{~g}$ for $20 \mathrm{~min}$ at $4^{\circ} \mathrm{C}$. Then, $100 \mu \mathrm{L}$ of supernatant was moved to a new microtube, and 200 $\mu \mathrm{L}$ of methanol was added to it. After 2 minutes of vortexing, the samples followed the producer same as a plasma sample.

\subsection{Statistical data analysis}

In this study, all data were presented as mean \pm standard deviation. The responses obtained from experiments were analyzed using the software Mini Table 18. Also, the possible mathematic models were analyzed using ANOVA one way. The best-fitting model was selected for each response-based on $P$ values. Also, $P$ values of $<0.05$ were considered as statistically significant. All figures were sketched using the Microsoft Excel version 2016.

The experiments were conducted in triplicate with the obtained results presented as a mean \pm standard deviation.

\section{Result And Discussion}

3.1. M.D. optimization in synthesis CLN and CH-CLN 
An M.D. with three independent variables at three different levels was used to study the response of the dependent variable to the experiments. Three different variables, namely Span 60, Chol, and Tween 60 (Table 1), were screened using M. D., and seven different Cn niosome samples were then achieved, as shown in Table 2.

\subsubsection{Fitting of data to the model}

The Fitted data obtained from responses to different models are presented in Table3 showing that the cubic model was the most compatible model for all the dependent variables.

Table 3

Summary of regression analysis for responses: Y1 (particle Size in nm), Y2 (encapsulation efficiency in \%), Y3 (PDI), and Y4 (zeta potential) for niosome samples.

\begin{tabular}{|c|c|c|c|c|c|c|c|c|c|c|c|c|}
\hline \multirow[t]{3}{*}{ parameter } & \multicolumn{12}{|l|}{ Model } \\
\hline & \multicolumn{3}{|c|}{ Response (Y1) } & \multicolumn{3}{|c|}{ Response (Y2) } & \multicolumn{3}{|c|}{ Response (Y3) } & \multicolumn{3}{|c|}{ Response (Y4) } \\
\hline & Linear & Quadratic & Cubic & Linear & Quadratic & Cubic & Linear & Quadratic & Cubic & Linear & Quadratic & Cubic \\
\hline $\begin{array}{l}\text { Adjusted } \\
\mathrm{R}^{2}\end{array}$ & 0.814 & 0.881 & 0.925 & 0.874 & 0.904 & 0.963 & 0.883 & 0.925 & 0.981 & 0.758 & 0.806 & 0.875 \\
\hline $\mathrm{R}^{2}$ & 0.817 & 0.885 & 0.929 & 0.877 & 0.907 & 0.969 & 0.890 & 0.930 & 0.985 & 0.760 & 0.810 & 0.879 \\
\hline
\end{tabular}

3.1.2. The effect of experimental variables on particle size

The particle sizes were within the range of $70-153 \mathrm{~nm}$, which are shown in Table 2. The results indicate that the Chol concentration has significantly affected the niosome size, in agreement with previous findings, which showed that niosome particle size has linearly increased along with the Chol concentration (Goyena and Fallis 2019). The results also revealed that particle size has been obviously augmented by increasing the span60: tween60 ratio, which is in agreement with the reports published by Rajabzadeh (2017)(Basiri et al. 2017a), Lee (2005) (Lee et al. 2005), and Essa(2010)(Essa 2010).

Figure $1(a, b, c)$ illustrate the effects of span 60 , Chol, and tween 60 concentrations on the particle size.

At a low Chol concentration, the shell is more flexible and pliable to the consequences of ultrasound waves. Hence, by increasing the Chol amount, the shell rigidity and ultrasound power resistance increase; and niosome with larger particle size would be produced(Nasseri 2005).

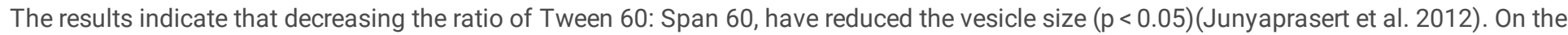
other hand, the average volume sizes of the niosomes decreased by increasing the HLB value(Basiri et al. 2017a). In this regard, the previous results have also shown that surfactant with long alkyl chain and small head group increased the niosome size(Basiri et al. 2017a). Moreover, smaller critical packing parameter (CPP) and higher hydrophilicity of the Tween 60 were known as the main reasons for this phenomena. As shown in Fig. 3(a, b, c), a significant entire CPP value for vesicle formation was also obtained at the smallest amount of Chol(Manosroi et al. 2010).

\subsubsection{Effect of variables on EE}

The EE of CLNs was between 33\% (NHM810) and 90\% (NHM840). in order to find the relationship between the independent factors and the EE of niosomes, Minitab 18 was used.

As reported by Nadzir (2018), the EE is correlated with the particle size, since the entrapment of Cn increased the vesicle diameter (Nadzir 2018).

The effects of variable concentrations on EE are shown in Table 2 and Fig. $1(d, e, f)$. The encapsulation efficiency was linearly increased by increasing the Chol content, due to the membrane stability brought by Chol(Hayashi et al. 2011). This data is in agreement with the results previously reported by Basiri(Basiri et al. 2017a) and Rinald(Kassem et al. 2017).

Another important critical parameter affecting the EE is surfactant characteristics such as alkyl chain length and surfactant content. It was indicated that the surfactant ability to form the vesicle mostly depends on the balance between hydrophobic and hydrophilic portions and surfactant structure (Noronha et al. 2013). In line with previous studies, the lower the HLB of the surfactant, the more reduction in EE of Cn(Seleci et al. 2016).

3.1.4. The effect of variables on PDI 
The different formulations ranged from 0.152 to 0.371 , indicating narrow distribution and relative homogeneity of the vesicle size. Also, PDI increased by decreasing the HLB value(Sadeghi Ghadi et al. 2019). Accordingly, the high PDI value in the case of NHM840 could be attributed to the agglomeration problems. Figure $1(\mathrm{~g}, \mathrm{~h}, \mathrm{i})$ reflects the variations in the size distribution dependency to the Chol content. The results indicate that the higher value of the PDI belonged to the sample, which had a higher Chol content.

\subsubsection{The effect of variables on Zeta potential}

Zeta potential values of different samples from -23 to $-30 \mathrm{mV}$ in Fig. 1 show that reduction of the HLB value means an rise in hydrophobicity, and then zeta potential increases. Also the surfactant type might affect the zeta potential (Sadeghi Ghadi et al. 2019).

\subsubsection{Optimization by M.D.}

The MD defines the relationship between independent and dependent variables. In addition, the multiple response optimizations were carried out to predict three set levels of experimental conditions by the minimum particle size and PDI, maximum EE, and optimum zeta potential. In this study, the maximum EE was predicted to be obtained at a combined level of $0.85(\mathrm{~g}), 0.219(\mathrm{~g})$, and 0.0953 (g) of Span 60, Tween 60, and Chol, respectively (Fig. 2). EE has linearly increased by increasing the HLB; and PDI, size, and zeta potential decreased by increasing the HLB value. These findings are in the relevant agreement with the previous studies(Basiri et al. 2017b). Also, it was found that in the range of set levels of the main preparation conditions, all seven combinations showed acceptable particle sizes (70-153 nm).

\subsection{Fourier Transform Infrared (FT-IR) spectroscopy}

FT-IR spectra were performed in order to consider the functional groups and their possible interactions in the prepared niosome structure. As shown in Fig. 3, FT-IR spectrum of Chol indicates the characteristic peak of hydroxyl stretching at $3400 \mathrm{~cm}^{-1}$, and aliphatic $\mathrm{C}-\mathrm{H}$ stretching in the region between 2800 and $2990 \mathrm{~cm}^{-1}$. The characteristic peaks located at 2918 and $2851 \mathrm{~cm}^{-1}$ in Tween $60 \mathrm{spectrum} \mathrm{(Fig.} 3$, c) were shown to be related to asymmetric and symmetric vibrations of methylene $(-\mathrm{CH} 2)$ groups, respectively.

The formation of niosomes was proven with two characteristic peaks at 1737 and $1106 \mathrm{~cm}^{-1}$ (Fig. 3, a, f), which are corresponded to vibrations of $\mathrm{C}=\mathrm{O}$ stretching, and C-O-C deformation(Sharma et al. 2015a) (Waddad et al. 2013).

In $\mathrm{CH}-\mathrm{CLNs}$, the absorption peak at $1415 \mathrm{~cm}^{-1}$ in $\mathrm{CH}-\mathrm{CLNs}$ can be attributed to the chitosan $\mathrm{OH}$ bending (Fig. 3. $\mathrm{g}$ ). Moreover, the $\mathrm{C}--\mathrm{O}--\mathrm{H}$ deformation peak of CLN at 1106 and $1045 \mathrm{~cm}^{-1}$ were shifted to 1111 and $1051 \mathrm{~cm}^{-1}$ in $\mathrm{CH}-\mathrm{CLN}$, respectively, due to the formation of the hydrogen bonds between chitosan and niosome.

Figure $3 \mathrm{~b}$ illustrates the spectrum of free $\mathrm{Cn}$. Also, the bands at 3508,1510 , and $1154 \mathrm{~cm}^{-1}$ are attributed to $\mathrm{O}-\mathrm{H}$ stretching, aromatic ring $\mathrm{C}=$ C stretching, and C-O stretching, respectively(Alemi et al. 2018).

Furthermore, the broader bands at $3500 \mathrm{~cm}^{-1}$ for CLN confirmed $\mathrm{Cn}$ entrapment in the vesicle system(Alemi et al. 2018) compared to the unloaded niosomes.

\subsection{CLN and CH-CLN Sizes, morphologies and zeta potential}

DLS, TEM, and AFM were used to consider their sizes and morphologies. The TEM and AFM images of the optimum CLN and CH-CLN are shown in Fig. 4.

A large particle size in the AFM images can be attributed to the vesicle aggregation that occurred during the air-drying production of the sample preparation. To evaluate the AFM validity result, $\mathrm{CH}-\mathrm{CLN}$ and CLN were supplementary investigated using TEM. A relatively spherical shaped morphology for the vesicles was demonstrated by the TEM picture as well, exhibiting a unilamellar membrane for the prepared CLN and $\mathrm{CH}-\mathrm{CLN}$. Notably, no aggregates were observed for the prepared samples in TEM(Zou et al. 2014).

The vesicle diameters determined from DLS, AFM, and TEM are illustrated in Table 5. Besides, particle sizes observed from AFM and TEM images were within the range of $40-100 \mathrm{~nm}$. These results are in agreement with the DLS results, which are more precise because the number of the analyzed particle by AFM and TEM were smaller than DLS(Rinaldi et al. 2018).

The particle size of $\mathrm{CH}-\mathrm{CLN}$ was more prominent than CLN, suggesting the formation of an extra chitosan layer on the CLN surface(Guo et al. 2003).

It is clear from Fig. 4 that niosome coated with chitosan shifted the zeta potential from negative $(-31 \mathrm{mV})$ to positive values $(+35 \mathrm{mV})$. The increase in zeta potential was attributed to the electrostatic interaction between negatively charged of surfactant and positively charged amino groups of chitosan According to literature, Positive zeta potential value was demonstrated the incomplete surface coating by chitosan (Ezzat et al. 2019).

\subsection{Cn Release Study}

Page $7 / 18$ 
In this study, the optimum samples were used to study the release behavior in the SGF and SIF. The cumulative release profile of the niosomes is shown in Fig. 5 (I). Accordingly, the results reveale that CLN and $\mathrm{CH}-\mathrm{CLN}$ released $50 \%$ and $35 \%$ of $\mathrm{Cn}$ within 8 h, respectively. Moreover, both of the CLN and $\mathrm{CH}-\mathrm{CLN}$ exhibited a sustained release profile.

Four different kinetic models were used to analyze the mode of release (Gautam Singhvi 2011)'(Sadati Behbahani et al. 2019) as follows: zero-order, first-order, Higuchi, and Hixon- Crowell models. (Table 4). Figure 5(II) shows the different model releases of CLNs and CH-CLN.

Comparing the four equations reported in Table 4 and Fig. 5(II), it can be said that the release of Cn followed the Hixson- Crowell model, due to the higher correlation coefficient $\left(\mathrm{R}^{2}\right)$. Furthermore, the model of Hixcon-Crowell was based on the corrosion mechanism, which explains that $\mathrm{Cn}$ release is a corrosion -controlled model(Xu et al. 2019). Overall, it can be reported that the chitosan, which coated the niosome surface, affected the way the drug was released, because it has an interaction with release media such as salt and biological fluid, leading to a better control of drug release.

\subsection{Distributions of Cn, CLN, and CH-CLN in organs after i.p. injection}

\subsubsection{HPLC analysis and method validation}

The HPLC calibration curve for plasma, and different regions of the brain and liver showed an excellent linearity $\left(R^{2}>0.995\right)$ over the ranges of $0.4,0.8,1.2,1.6$, and $2 \mu \mathrm{g} / \mathrm{ml}$. Also, the LOD (limit of detection) and LOQ (limit of quantification) Cn were obtained as 0.0186 and 0.0565 $\mu \mathrm{g} / \mathrm{ml}$, respectively. Cn inter assay and intra assay precision, accuracy, and precision value in the brain, plasma, and liver were $15 \%$. Altogether, these results show the validity of the method in this study.

3.6.2 In vivo study

As shown in Fig. 6, the concentrations of $\mathrm{Cn}$ have significantly increased in plasma, liver, and brain regions when loaded in niosomes nanoparticles, compared to free $\mathrm{Cn}$, which indicate the improved stability and delivery of CLN and CH-CLN.

It is noteworthy that, there were some crucial differences in Cn existence in the brain, liver, and plasma. Cn, in its natural form, was not detected in the above-mentioned organs. Also, a difference between $\mathrm{Cn}$ and nanocarrier can be attributed to the resistance to liver metabolism(Tsai et al. 2011),(Raza et al. 2017) and BBB nature.

The BBB is a protective reticulum around the brain that only allows the penetration of low molecular weight and/or small molecules with a high lipid solubility. Thus, passing BBB seems difficult for curcumin. In nanocarrier, the appropriate size and existence of tween 60 in niosome structure could act as an apolipoprotein E anchor in the bloodstream, which can consequently improve passing BBB(Hombach and BernkopSchnürch 2009). The vesicles can also interact with the LDL receptor and pass the BBB(Goyena and Fallis 2019).

The $\mathrm{Cn}$ distribution and concentration in rat organs were increased by modifying the niosome surface. The results demonstrate that $\mathrm{Cn}$ concentration was significantly higher in different regions of the central nervous system of rats that received CH-CLN compared to CLN: 16 $(0.019 ; 0.001 \mathrm{mg} / \mathrm{Kg})$ in the striatum, $16(0.019 ; 0.001 \mathrm{mg} / \mathrm{Kg})$ in the cerebellum, and $3.5(0.320 ; 0.009 \mathrm{mg} / \mathrm{Kg})$ in the cerebral cortex. Accordingly, it suggests that positively charged chitosan-coated niosome can cooperate with all the negatively charged elements in a biological milieu, which consequently increases $\mathrm{CH}-\mathrm{CLN}$ permeation ability[50,51].

Cn has a rapid degradation under physiological condition, so its encapsulation in niosome and chitosan-coated niosome may hinder its degradation. Also, in the case of $\mathrm{CH}-\mathrm{CLN}, \mathrm{Cn}$ concentration in rat plasma has reduced more gradually compared to the CLN form, due to the protective effect of chitosan layer(Hombach and Bernkop-Schnürch 2009)(Akhtar et al. 2012). It has been previously reported that chitosan. (Akhtar et al. 2012), human serum albumin(Leung and Kee 2009), and aS1-Casein(Sneharani et al. 2009) could improve stability of Cn in different carrier systems. Furthermore, the highest amount of $\mathrm{Cn}$ in both groups was detected in the cerebral cortex, because the $\mathrm{Cn}$ disposition was affected by brain cell transport(Tsai et al. 2011).

\section{Conclusion}

In the present study, CLN and CH-CLN were successfully synthesized using a modified heating method. All the niosomes were $<200$ nm in diameter. Also, M.D. was used to optimize the niosome components to achieve the minimum vesicle size and a high EE. Then, chitosan was utilized to modify the optimal niosome surface.

Free $\mathrm{Cn}, \mathrm{CLN}$, and $\mathrm{CH}-\mathrm{CLN}$ were i. p. administrated on Wistar rat and the presence of $\mathrm{Cn}$ in the brain, plasma, and liver was investigated utilizing HPLC. The concentration of $\mathrm{Cn}$ in brain and plasma, which was administered with $\mathrm{CH}-\mathrm{CLN}$, was higher than those that were treated with CLN. Also, CH-CLN increased the BBB permeability and bloodstream stability of $\mathrm{Cn}$ in comparison with CLN. The results confirm the efficacy of chitosan on the improvement of niosome, as the brain target delivery candidate.

Page $8 / 18$ 


\section{Declarations}

\section{Acknowledgments}

The authors would like to acknowledge the Department of Food Nanotechnology, Research Institute of Food Science and Development, Curtin University Malaysia, for their constant support during this study. Neuroscience Research center, Mashhad University of Medical Sciences.

\section{Ethics declarations}

Ethics approval All procedures were conducted in accordance with the accepted principles for the care and use of laboratory animals and were approved by the animal ethics committee of Mashhad University of Medical Sciences (IR.mums.Rec.1399.287).

\section{Consent to participate}

\section{Author's contribution}

SS carried out all the experiments and prepared the manuscript. MY helped in preparing the paper. SSN consulted SS during the animal study. GhR and MSN supervised and conceived the whole study, and edited the paper. The authors declare that all data were generated in-house and that no paper mill was used. All authors read and approved the manuscript. The authors declare that all data were generated in-house and that no paper mill was used.

\section{Funding}

This research received no specific grant from funding agencies in the public, commercial, or not-for-profit sectors.

\section{Conflict of interest}

The authors declare that they have no conflict of interest.

\section{Consent to publish}

Not applicable

\section{References}

1. Akhtar F, Rizvi MMA, Kar SK (2012) Oral delivery of curcumin bound to chitosan nanoparticles cured Plasmodium yoelii infected mice. Biotechnol Adv 30:310-320. https://doi.org/10.1016/j.biotechadv.2011.05.009

2. Alemi A, Zavar Reza J, Haghiralsadat $F$ et al (2018) Paclitaxel and curcumin coadministration in novel cationic PEGylated niosomal formulations exhibit enhanced synergistic antitumor efficacy. J Nanobiotechnology 16:1-20. https://doi.org/10.1186/s12951-018-03514

3. Basiri L, Rajabzadeh G, Bostan A (2017a) a-Tocopherol-loaded niosome prepared by heating method and its release behavior. Food Chem 221:620-628. https://doi.org/10.1016/j.foodchem.2016.11.129

4. Basiri L, Rajabzadeh G, Bostan A (2017b) Physicochemical properties and release behavior of Span 60/Tween 60 niosomes as vehicle for a-Tocopherol delivery. LWT - Food Sci Technol 84:471-478. https://doi.org/10.1016/j.lwt.2017.06.009

5. Chirio D, Peira E, Sapino S et al (2018) Stearoyl-chitosan coated nanoparticles obtained by microemulsion cold dilution technique. Int J Mol Sci 19:. https://doi.org/10.3390/ijms19123833

6. Dalvi AV, Uppuluri CT, Bommireddy EP, Ravi PR (2018) Design of experiments-based RP - HPLC bioanalytical method development for estimation of Rufinamide in rat plasma and brain and its application in pharmacokinetic study. Elsevier B.V

7. Essa EA (2010) Effect of formulation and processing variables on the particle size of sorbitan monopalmitate niosomes. https://doi.org/10.4103/0973-8398.76752

8. Ezzat HM, Elnaggar YSR, Abdallah OY (2019) Improved oral bioavailability of the anticancer drug catechin using chitosomes: Design, invitro appraisal and in-vivo studies. Int J Pharm 565:488-498. https://doi.org/10.1016/j.ijpharm.2019.05.034

9. Gautam Singhvi MS (2011) In-vitro drug release characterization models. Int J Pharm Stud Res II:77-84

10. Goyena R, Fallis A (2019) Proniosomes as a Stable Carrier for Oral Acyclovir: Formulation and Physicochemical Characterization Viviane. J Chem Inf Model 53:1689-1699. https://doi.org/10.1017/СВ09781107415324.004

11. Guo J, Ping Q, Jiang G et al (2003) Chitosan-coated liposomes: Characterization and interaction with leuprolide. Int J Pharm 260:167173. https://doi.org/10.1016/S0378-5173(03)00254-0 
12. Hayashi K, Shimanouchi T, Kato K et al (2011) Colloids and Surfaces B: Biointerfaces Span 80 vesicles have a more fluid, flexible and “ wet " surface than phospholipid liposomes. Colloids Surfaces B Biointerfaces 87:28-35. https://doi.org/10.1016/j.colsurfb.2011.04.029

13. Hoemann CD, Guzmán-Morales J, Tran-Khanh N et al (2013) Chitosan rate of uptake in HEK293 cells is influenced by soluble versus microparticle state and enhanced by serum-induced cell metabolism and lactate-based media acidification. Molecules 18:1015-1035. https://doi.org/10.3390/molecules18011015

14. Hombach J, Bernkop-Schnürch A (2009) Chitosan solutions and particles: Evaluation of their permeation enhancing potential on MDCK cells used as blood brain barrier model. Int J Pharm 376:104-109. https://doi.org/10.1016/j.ijpharm.2009.04.027

15. Hoppe JB, Coradini K, Frozza RL et al (2013) Free and nanoencapsulated curcumin suppress $\beta$-amyloid-induced cognitive impairments in rats: Involvement of BDNF and Akt/GSK-3 $\beta$ signaling pathway. Neurobiol Learn Mem 106:134-144. https://doi.org/10.1016/j.nlm.2013.08.001

16. Junyaprasert VB, Singhsa P, Suksiriworapong J, Chantasart D (2012) Physicochemical properties and skin permeation of Span 60/Tween 60 niosomes of ellagic acid. Int J Pharm 423:303-311. https://doi.org/10.1016/j.ijpharm.2011.11.032

17. Kassem MA, El-Sawy HS, Abd-Allah FI et al (2017) Maximizing the Therapeutic Efficacy of Imatinib Mesylate-Loaded Niosomes on Human Colon Adenocarcinoma Using Box-Behnken Design. J Pharm Sci 106:111-122. https://doi.org/10.1016/j.xphs.2016.07.007

18. Khalifa AZM, Abdul Rasool BK (2017) Optimized Mucoadhesive Coated Niosomes as a Sustained Oral Delivery System of Famotidine. AAPS PharmSciTech 18:3064-3075. https://doi.org/10.1208/s12249-017-0780-7

19. Khallaf RA, Aboud HM, Sayed OM (2019) Surface modified niosomes of olanzapine for brain targeting via nasal route; preparation, optimization, and in vivo evaluation. J Liposome Res 0:1-11. https://doi.org/10.1080/08982104.2019.1610435

20. Le TT, Kim D (2019) Folate-PEG/Hyd-curcumin/C18-g-PSI micelles for site specific delivery of curcumin to colon cancer cells via Wnt/ $\beta$ catenin signaling pathway. Mater Sci Eng C 101:464-471. https://doi.org/10.1016/j.msec.2019.03.100

21. Lee S, Lee K, Kim J, Lim S (2005) The Effect of Cholesterol in the Liposome Bilayer on the Stabilization of Incorporated Retinol. $157-166$. https://doi.org/10.1080/08982100500364131

22. Leung MHM, Kee TW (2009) Effective stabilization of curcumin by association to plasma proteins: Human serum albumin and fibrinogen. Langmuir 25:5773-5777. https://doi.org/10.1021/la804215v

23. Li R, Deng L, Cai Z et al (2017) Liposomes coated with thiolated chitosan as drug carriers of curcumin. Mater Sci Eng C 80:156-164. https://doi.org/10.1016/j.msec.2017.05.136

24. Manosroi A, Khanrin P, Lohcharoenkal W et al (2010) Transdermal absorption enhancement through rat skin of gallidermin loaded in niosomes. Int J Pharm 392:304-310. https://doi.org/10.1016/j.ijpharm.2010.03.064

25. Mészáros M, Porkoláb G, Kiss L et al (2018) Niosomes decorated with dual ligands targeting brain endothelial transporters increase cargo penetration across the blood-brain barrier. Eur J Pharm Sci 123:228-240. https://doi.org/10.1016/j.ejps.2018.07.042

26. Moghddam SRM, Ahad A, Aqil M et al (2016) Formulation and optimization of niosomes for topical diacerein delivery using 3-factor, 3level Box-Behnken design for the management of psoriasis. Mater Sci Eng C 69:789-797. https://doi.org/10.1016/j.msec.2016.07.043

27. Mozafari MR, Johnson C, Hatziantoniou S, Demetzos C (2008) Nanoliposomes and their applications in food nanotechnology. J Liposome Res 18:309-327. https://doi.org/10.1080/08982100802465941

28. Mythri RB, Jagatha B, Pradhan N et al (2007) Mitochondrial complex I inhibition in Parkinson's disease: How can curcumin protect mitochondria? Antioxidants Redox Signal 9:399-408. https://doi.org/10.1089/ars.2006.1479

29. Nadzir MM (2018) Effects of cholesterol and charging additives on stability of curcumin niosomes. https://doi.org/10.3923/jeasci.2017.8537.8541

30. Nasseri B (2005) Effect of cholesterol and temperature on the elastic properties of niosomal membranes. 300:95-101. https://doi.org/10.1016/j.ijpharm.2005.05.009

31. Noronha CM, Granada AF, de Carvalho SM et al (2013) Optimization of a-tocopherol loaded nanocapsules by the nanoprecipitation method. Ind Crops Prod 50:896-903. https://doi.org/10.1016/j.indcrop.2013.08.015

32. Rafiee Z, Nejatian M, Daeihamed M, Jafari SM (2018) Application of different nanocarriers for encapsulation of curcumin. Crit Rev Food Sci Nutr 0:1-30. https://doi.org/10.1080/10408398.2018.1495174

33. Raza K, Kumar P, Kumar N, Malik R (2017) Pharmacokinetics and biodistribution of the nanoparticles. Adv Nanomedicine Deliv Ther Nucleic Acids 166-186. https://doi.org/10.1016/B978-0-08-100557-6.00009-2

34. Rinaldi F, Hanieh PN, Chan LKN et al (2018) Chitosan glutamate-coated niosomes: A proposal for nose-to-brain delivery. Pharmaceutics 10:1-16. https://doi.org/10.3390/pharmaceutics10020038

35. Rinaldi F, Hanieh PN, Imbriano A et al (2020) Different instrumental approaches to understand the chitosan coated niosomes/mucin interaction. J Drug Deliv Sci Technol 55:101339. https://doi.org/10.1016/j.jddst.2019.101339

Page $10 / 18$ 
36. Ringman JM, Frautschy SA, Teng E et al (2012) Oral curcumin for Alzheimer's disease: Tolerability and efficacy in a 24-week randomized, double blind, placebo-controlled study. Alzheimer's Res Ther 4:43. https://doi.org/10.1186/alzrt146

37. Sadati Behbahani E, Ghaedi M, Abbaspour M et al (2019) Curcumin loaded nanostructured lipid carriers: In vitro digestion and release studies. Polyhedron 164:113-122. https://doi.org/10.1016/j.poly.2019.02.002

38. Sadeghi Ghadi Z, Dinarvand R, Asemi N et al (2019) Preparation, characterization and in vivo evaluation of novel hyaluronan containing niosomes tailored by Box-Behnken design to co-encapsulate curcumin and quercetin. Eur J Pharm Sci 130:234-246.

https://doi.org/10.1016/j.ejps.2019.01.035

39. Sarvaiya J, Agrawal YK (2015) Chitosan as a suitable nanocarrier material for anti-Alzheimer drug delivery. Int J Biol Macromol 72:454465. https://doi.org/10.1016/j.ijbiomac.2014.08.052

40. Seleci DA, Seleci M, Walter J et al (2016) Niosomes as Nanoparticular Drug Carriers: Fundamentals and Recent Applications. 2016

41. Sharma V, Anandhakumar S, Sasidharan M (2015a) Self-degrading niosomes for encapsulation of hydrophilic and hydrophobic drugs: An efficient carrier for cancer multi-drug delivery. Mater Sci Eng C 56:393-400. https://doi.org/10.1016/j.msec.2015.06.049

42. Sharma V, Anandhakumar S, Sasidharan M (2015b) Self-degrading niosomes for encapsulation of hydrophilic and hydrophobic drugs: An efficient carrier for cancer multi-drug delivery. Mater Sci Eng C 56:393-400. https://doi.org/10.1016/j.msec.2015.06.049

43. Sneharani AH, Singh SA, Rao AGA (2009) Interaction of aS1-casein with curcumin and its biological Implications. J Agric Food Chem 57:10386-10391. https://doi.org/10.1021/jf902464p

44. Tiwari SK, Agarwal S, Seth B et al (2014) Curcumin-loaded nanoparticles potently induce adult neurogenesis and reverse cognitive deficits in Alzheimer's disease model via canonical Wnt/ $\beta$-catenin pathway. ACS Nano 8:76-103. https://doi.org/10.1021/nn405077y

45. Tsai YM, Chien CF, Lin LC, Tsai TH (2011) Curcumin and its nano-formulation: The kinetics of tissue distribution and blood-brain barrier penetration. Int J Pharm 416:331-338. https://doi.org/10.1016/j.ijpharm.2011.06.030

46. Waddad AY, Abbad S, Yu F et al (2013) Formulation, characterization and pharmacokinetics of Morin hydrate niosomes prepared from various non-ionic surfactants. Int J Pharm 456:446-458. https://doi.org/10.1016/j.ijpharm.2013.08.040

47. Xu W, Huang L, Jin W et al (2019) Encapsulation and release behavior of curcumin based on nanoemulsions-filled alginate hydrogel beads. Int J Biol Macromol 134:210-215. https://doi.org/10.1016/j.ijbiomac.2019.04.200

48. Xu Y-Q, Li P, Wan J-B et al (2016) Niosome encapsulation of curcumin: Characterization and cytotoxic effect on ovarian cancer cells. J Nanomater 2016:0-9. https://doi.org/10.1155/2016/6365295

49. Yallapu MM, Jaggi M, Chauhan SC (2012) Curcumin nanoformulations: a future nanomedicine for cancer. Drug Discov Today 17:71-80. https://doi.org/10.1016/j.drudis.2011.09.009

50. Yang KY, Lin LC, Tseng TY et al (2007) Oral bioavailability of curcumin in rat and the herbal analysis from Curcuma longa by LC-MS/MS. J Chromatogr B Anal Technol Biomed Life Sci 853:183-189. https://doi.org/10.1016/j.jchromb.2007.03.010

51. Zou L, Peng S feng, Liu W et al (2014) Improved in vitro digestion stability of (-)-epigallocatechin gallate through nanoliposome encapsulation. Food Res Int 64:492-499. https://doi.org/10.1016/j.foodres.2014.07.042

\section{Figures}



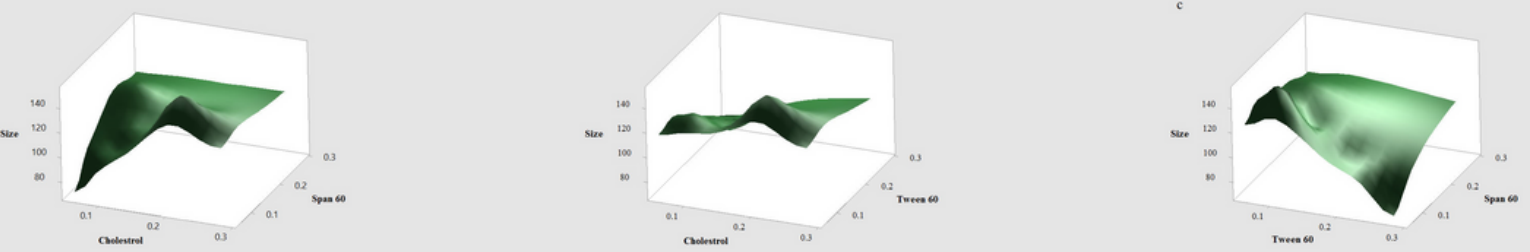

d
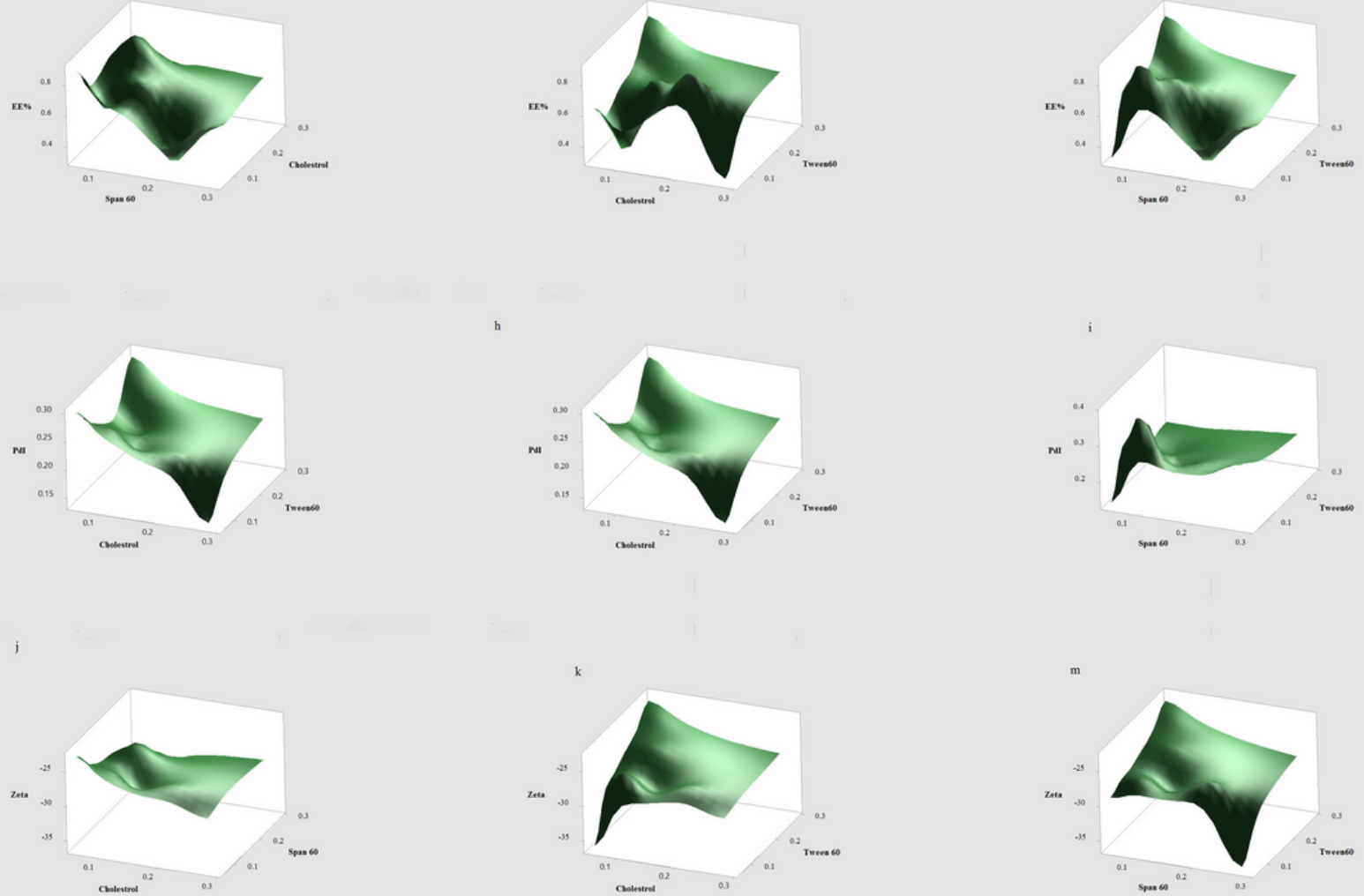

\section{Figure 1}

Three-dimensional response surface plot presenting the effect of independent variables on niosomes: particle size (a, b, c), EE (d, e, f) PDI (g, $h, i)$, and zeta potential $(j, k, m)$. 


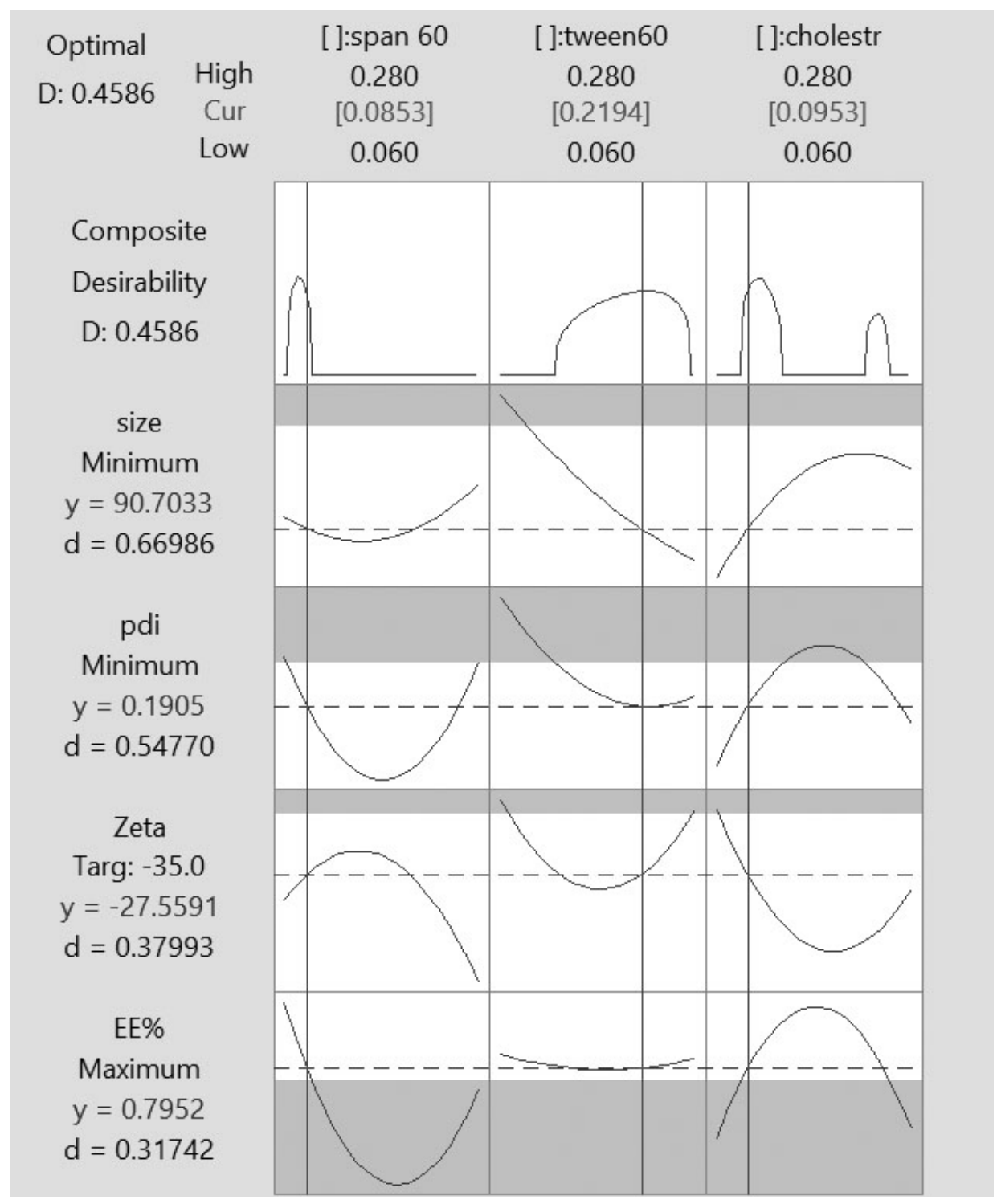

\section{Figure 2}

Response optimization, parameters, predicted responses, and desirability. 


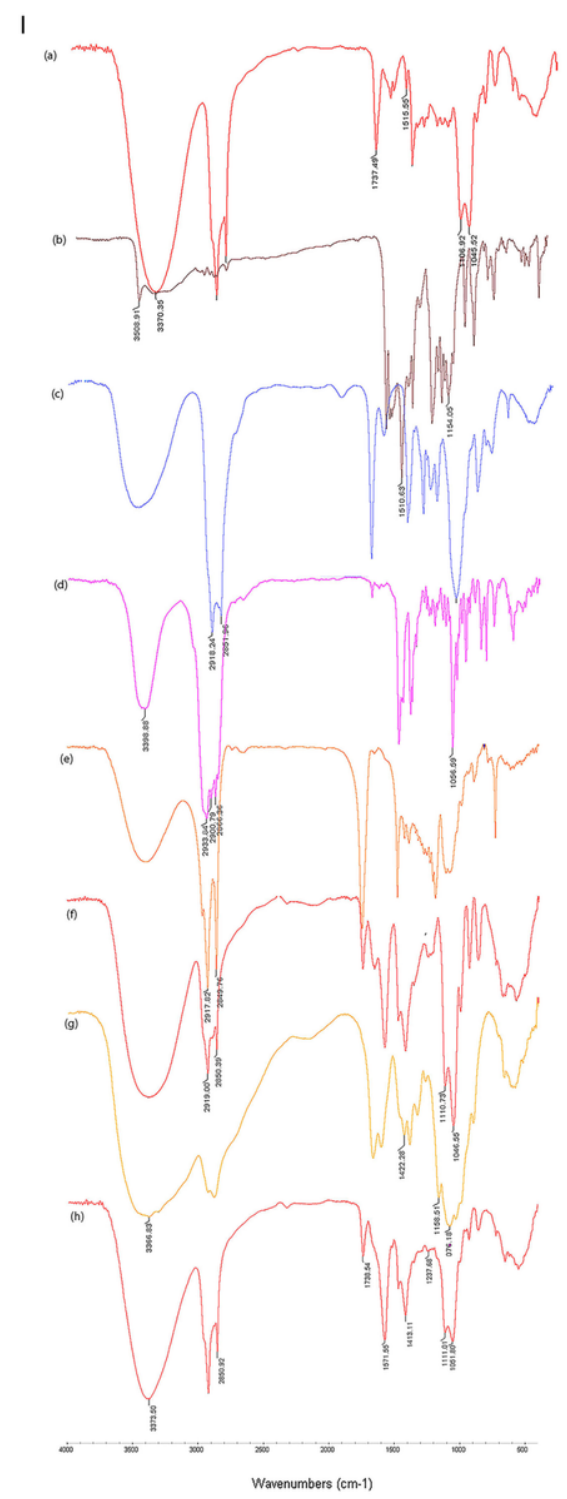

Figure 3

FT-IR spectra of (a) CLN, (b) Cn, (c) Tween 60, (d) cholesterol, (e) Span60, (f) unloaded niosome (g) chitosan, and (h) CH-CLN. 

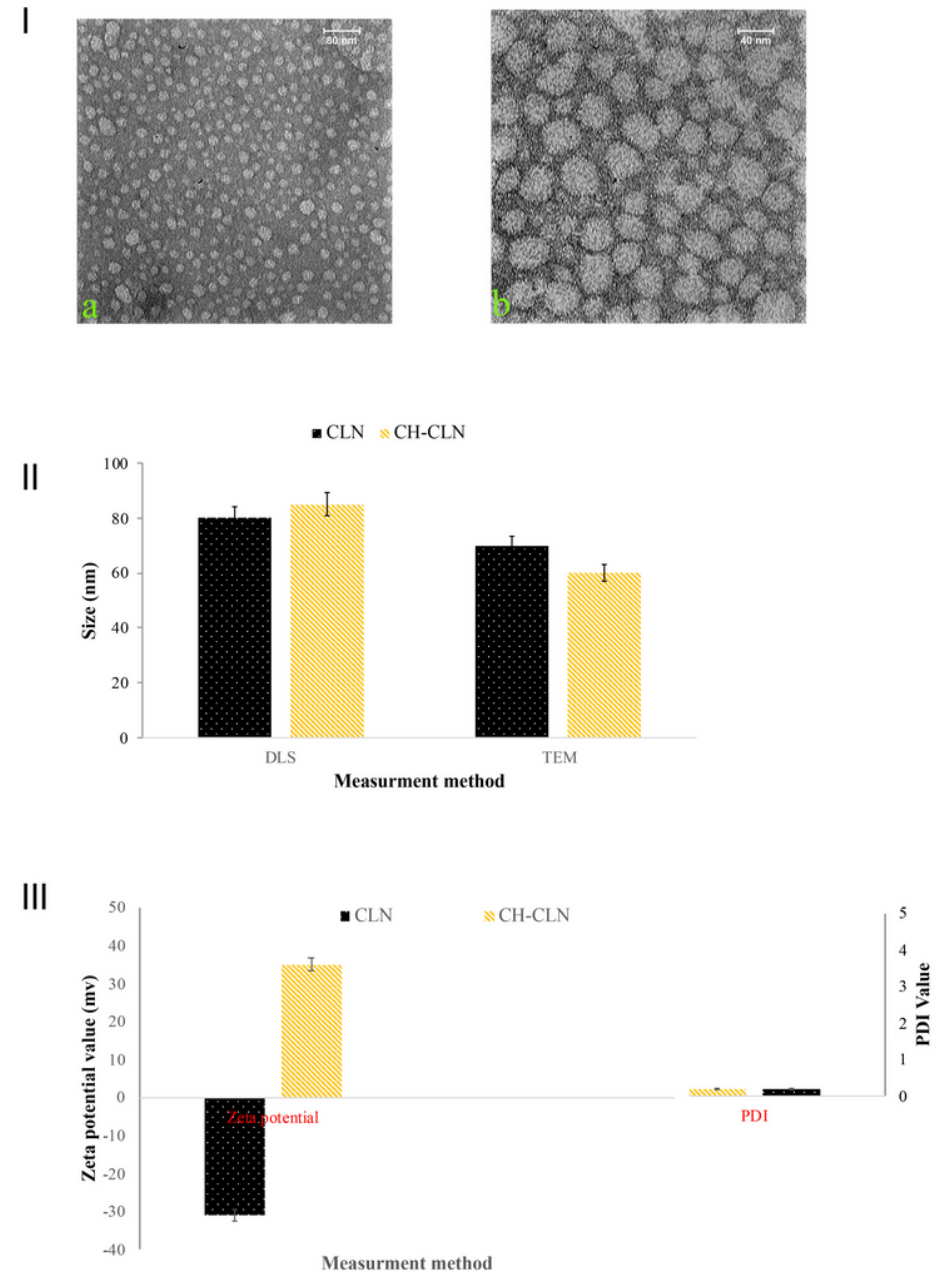

\section{Figure 4}

(I)TEM image of CLN (a) and CH-CLN (b). (II) Vesicle size of CLN and CH-CLN by DLS and TEM data. (III) Zeta potential and PDI value of CLN and $\mathrm{CH}-\mathrm{CLN}$. 

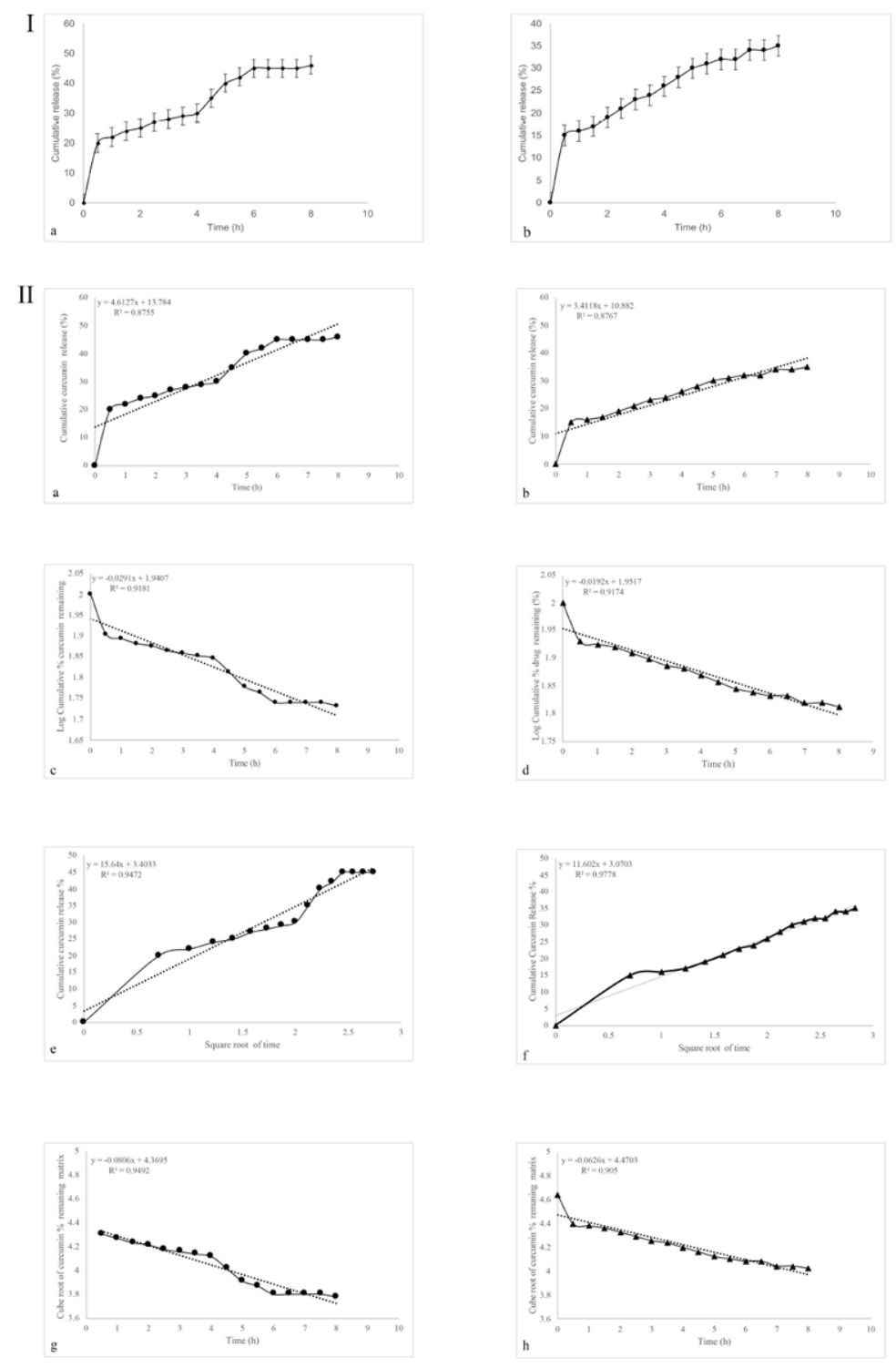

\section{Figure 5}

(I) The release profiles of CLN (a) and CH-CLN (b) in SGF and SIF.(II) Zero-order model (a, b), First-order model (c, d), Higuchi model (e, f), and Hixson-Crowell cube-root model(g, h) releases of CLN and CH-CLN respectively. 


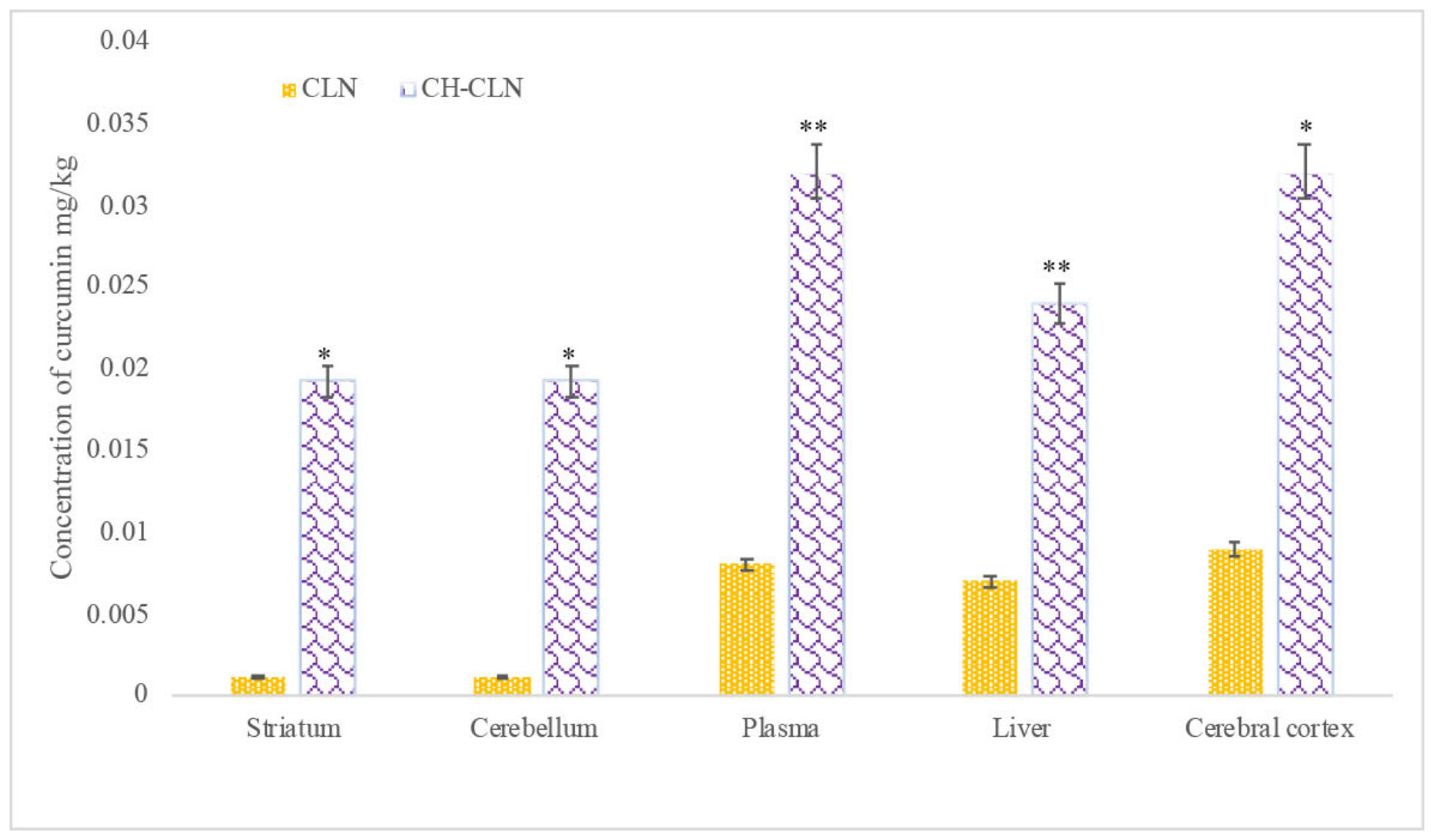

\section{Figure 6}

The concentrations of CLN and $\mathrm{CH}-\mathrm{CLN}$ in a different region of the central nervous system, liver, and plasma after 15 min administration (i.p. $50 \mathrm{mg} / \mathrm{kg}$ mean \pm SEM, $\mathrm{n}=4,{ }^{*} \mathrm{p}<0.05$ and $\left.{ }^{*} \mathrm{p}<0.001\right)$.

\section{Supplementary Files}

This is a list of supplementary files associated with this preprint. Click to download.

- CHcLN.tif

- CLN.csv

- CLN.tif

- ChCLN.csv

- Chitosan.csv

- Chitosan.tif

- SizePDImain.xIsx

- Span.csv

- TWee.csv

- TWee.tif

- barenioosme.csv

- barenioosme.tif

- cholestrol.csv

- cholestrol.tif

- curcum.csv

- curcum.tif

- graphsalehicopy.xIsx 
- $\mathrm{g}$

- release.xlsx

- span.tif

- supp1..xIsx

- tot.csv

- tot.xlsx

Page 18/18 